\title{
VARIATIONS DES ÉCHANGES RESPIRATOIRES EN FONCTION DE L'ÉTAT DE NUTRITION DES REINES DE BOURDONS. (Hymenoptera, Apoidea, Bombus Latr.)
}

\section{Änderungen des Gaswechsels bei der Atmung bei Hummelköniginnen in Abhängigkeit von ihrem Ernährungszustand.}

\author{
A. POUVREAU \\ Station de Recherches sur l'Abeille et les Insectes sociaux-I.N.R.A. \\ 91440 Bures sur Yvette
}

\section{SUMMARY}

VARIATIONS IN RESPIRATORY EXCHANGES

IN RELATION WITH THE NUTRITION STATE OF BUMBLE BEE QUEENS

The question of the influence of nutrition on the intensity of respiratory exchanges comes up in bumble bees during the period of activity, especially when they supply themselves abundantly with food reserves.

In this study the author attends to know, by measuring oxygen consumption, if regular feeding keeps up a regular respiratory activity and if fast alters the intensity of respiratory exchanges in bumble bee queens.

In order to avoid a too great heterogeneity in metabolic processes between the bumble bee queens, as well as a pollution of respirometric flask by elimination of feces, we subject insects to fast periods of 12,18 and 24 hours.

The experiments show that oxygen consumptions of bumble bee queens decrease gradually as the period of fast gets longer, in the limit of $24 \mathrm{~h}$ of experimentation.

The levels of oxygen consumption do not undergo a progressive decrease in relation with the duration of fast period. In the 4 studied species of Bombus (hypnorum, lapidarius, lucorum, pratorum), the results point out a clear lowering of oxygen consumption in queens subjected to a 12 hours long fast in comparison with control insects. The queens, which fasted during $18 \mathrm{~h}$, show values of respiratory exchanges lightly lower than those of previous exchanges. Then the means of oxygen consumption are submitted to a little more marked decrease in the queens subjected to a fast of 24 hours.

The decrease of respiratory intensity during the first hours of fast is usually in relation with a break in the digestion. The knowledge of the evolution of digestion is fundamental for the understanding of respiratory exchanges in the inanition period. 


\section{RÉSUMÉ}

La question de l'influence de la nutrition sur l'intensité des échanges respiratoires se pose chez les Bourdons en période d'activité, surtout lorsque ceux-ci se pourvoient abondamment de réserves.

Dans cette étude, l'auteur se préoccupe de savoir, au moyen de mesures de consommation d'oxygène, si une alimentation régulière entretient une activité respiratoire régulière, et si le jeûne modifie l'intensité des échanges respiratoires chez les reines de Bourdons.

Afin d'éviter une trop grande hétérogénéité au niveau des processus métaboliques entre les reines de Bourdons, ainsi qu'une pollution des fioles respirométriques par élimination de fèces, nous soumettons les Insectes à des périodes de jeûne de durées différentes (12 heures, 18 heures, 24 heures).

Les expériences montrent que les consommations d'oxygène des reines de Bombus diminuent graduellement à mesure que les durées de la période de jeûne augmentent, dans la limite de 24 heures de l'expérimentation.

Les niveaux de la consommation d'oxygène ne subissent pas une diminution progressive en fonction de la durée de la période de jeûne. Les résultats indiquent, chez les quatre espèces (hypnorum, lapidarius, lucorum, pratorum) de Bombus étudiées, un net abaissement de la consommation d'oxygène des reines soumises à un jeûne de 12 Heures par rapport aux Insectes témoins. Les reines ayant jeûné pendant 18 heures montrent des valeurs des échanges respiratoires légèrement inférieures à celles des échanges précédents. Puis, les moyennes de la consommation d'oxygène subissent une baisse un peu plus accentuée chez les reines soumises à un jeûne de 24 heures.

La diminution de l'intensité respiratoire au cours des premières heures de jeûne est, généralement, en relation avec une interruption de la digestion. La connaissance de l'évolution de cette dernière est donc fondamentale pour la compréhension des échanges respiratoires en phase d'inanition.

\section{INTRODUCTION}

La question de l'influence de la nutrition sur l'intensité des échanges respiratoires se pose chez les Insectes en période d'activité, surtout lorsque ceux-ci se pourvoient abondamment de réserves.

Pendant la période où les jeunes reines de Bourdons restent attachées à leur colonie maternelle, elles aident à l'approvisionnement du nid; puis, après s'être alimentées abondamment de nectar et de pollen, les reines accumulent des quantités importantes de substances de réserve dans leur tissu adipeux (Pouvreau, 1976).

En l'absence de toute variation des facteurs externes, les échanges respiratoires d'un même individu ne se maintiennent pas rigoureusement constants. Ils sont en effet soumis aux modifications de tout un ensemble d'éléments qui constituent l'état de l'Insecte à un moment donné.

Nous nous préoccupons de savoir, au moyen de mesures de consommation d'oxygène, si une alimentation régulière entretient une activité respiratoire régulière, et si le jeûne modifie l'intensité des échanges respiratoires chez des reines de différentes espèces de Bourdons.

\section{CONDITIONS EXPERIMENTALES}

\section{Matériel biologique}

Nos expériences portent sur les reines de quatre espèces de Bourdons (Pyrobombus (Pyrobombus) hypnorum L., Pyrobombus (Melanobombus) lapidarius L., Bombus (Bombus) lucorum L., Pyrobombus 
(Pyrobombus) pratorum L.), élevées en serre, où les conditions de lumière, de température et d'hygrométrie sont très voisines de celles qui règnent dans la nature. Pour permettre des comparaisons entre les différents états de nutrition, les reines soumises à une expérience ont le même âge, et proviennent de la même colonie.

Le maintien des reines de Bourdons en captivité durant tout leur cycle biologique, suivant une technique que nous avons mise progressivement au point (PouvreAu, 1976), nous permet une observation continue des Insectes et un prélèvement précis des reines soumises à l'expérimentation. En effet, entre chaque intervention expérimentale, les reines sont ramenées en serre, où elles ont la possibilité de retourner dans leur nid maternel.

Afin d'effectuer des répétitions de mesures respirométriques sur les mèmes femelles, celles-ci sont marquées, sur le scutum, au moyen d'une pastille de couleur portant un numéro.

Chaque série de mesures est pratiquée sur des reines de Bourdons en états de nutrition différents :

- reines prèlevées en serre et placées isolément dans les fioles du respiromètre. Ces Insectes se sont alimentés en serre, mais il n'est pas toujours possible de déterminer avec précision le niveau de nutrition atteint, lorsque la prise du dernier repas ne peut être constatée;

- reines en état de jeûne. Ces Insectes sont soumis à des durées de jeûne différentes : 12 heures, 18 heures, 24 heures.

Dans nos élevages, une alimentation abondante et variée est mise à la disposition des Bourdons. Une solution d'eau miellée ( $50 \%$ ) est distribuée dans des boites de Pétri remplies de billes de verre, afin d'éviter que les Insectes ne s'engluent. Des fleurs nectarifères et pollenifères, provenant de cultures et disposées en serre, permettent un approvisionnement en aliments frais. Du pollen est également fourni sous forme de pelotes, provenant de trappes à pollen de ruches d'Abeilles domestiques.

\section{Matériel technique}

Les mesures respirométriques sont effectuées à l'aide de l'appareil de Warburg. La méthode manométrique de Warburg se ramène, expérimentalement, à mesurer, à l'aide de manomètres, les variations de pression survenant dans chacune des fioles, et cela, à température et volume rigoureusement constants. Ces variations de pression sont ensuite converties en volumes ramenés aux conditions normales de température et de pression.

Le bain thermostatique est constitué par une cuve circulaire en plexiglas (capacité : 9 litres environ). Les manomètres sont disposés tout autour de la cuve. Le disque qui supporte les manomètres leur imprime un mouvement de va-et-vient autour de l'axe commun. Le brassage du liquide dans la cuve est assuré par une turbine. Pour éviter l'apparition de points chauds dans le bain, on rend l'eau conductrice par addition de chlorure de sodium, et on procède au chauffage par un système d'électrodes. L'élément thermosensible est constitué par un thermomètre à contact de 0 à $50^{\circ} \mathrm{C}$. Pour travailler à des températures voisines de la température ambiante ou inférieures, un serpentin de réfrigération de cryostat est utilisé.

Nous avons employé des manomètres différentiels à double capillaire, à volume constant (manomètres de Summerson). Ces manomètres présentent également la possibilité d’être utilisés comme des manomètres simples à double capillaire. Dans ce cas, l'une des branches d'un manomètre appelée thermobaromètre, sert de témoin et indique les variations de la pression atmosphérique, variations dont il convient de tenir compte.

Les fioles utilisées pour la consommation d'oxygène sont des fioles coniques, munies d'une ampoule latérale destinée à recevoir la solution d alcali (absorption du gaz carbonique), et comportant un puits central utilisable à diverses fins (adjonction d'eau, ...). L'agitation des fioles est indispensable pour assurer une température uniforme dans les fioles, une homogénéisation des gaz à l'intérieur de l'appareil, et une meilleure absorption du gaz carbonique par la solution de potasse.

L'ensemble de l'appareillage est disposé dans une pièce thermostatée, et dont l'hygrométrie correspond aux conditions d'humidité auxquelles sont soumis les Bourdons pendant leur vie active. La température de la pièce est réglée en fonction de la température expérimentale, de façon à ce que la partie du manomètre à l'air soit à la même température que la partie immergée dans le bain thermostatique. 


\section{Mode opératoire}

Chaque Insecte est prélevé à l'aide de pinces et placé dans un récipient pour être transporté et pesé. Après la pesée, la reine de Bourdon est transférée dans une fiole respirométrique. Afin de ne pas mettre en expérience un Insecte surexcité par les différentes manipulations dont il a été l'objet, la fiole est placée dans la salle "thermostatée ", en conditions de température et d'obscurité totale identiques aux conditions expérimentales.

Après une période d'adaptation d'une heure environ, la fiole est connectée au manomètre, puis immergée dans le bain thermostatique.

Le début des mesures respirométriques est précédé d'une période d'équilibration thermique d'environ une heure, au cours de laquelle il n'est pas possible de faire des mesures, car elles seraient entachées d'erreurs. Pendant cette période, l'Insecte consomme de l'oxygène et rejette du gaz carbonique. Ce dernier est absorbé par la solution de potasse, placée dans l'ampoule latérale de la fiole. L'atmosphère de la fiole, en communication avec l'air extérieur au moyen d'un robinet à trois voies, est renouvelée pendant la période d'équilibration thermique. La pression artificielle de l'oxygène dans la fiole est égale à celle de l'air atmosphérique.

La température de mesure des échanges respiratoires est de $19^{\circ} \mathrm{C}$.

La consommation d'oxygène est suivie simultanément pour sept reines appartenant à la mème espèce de Bombus, et soumises aux mêmes conditions de nutrition. Chaque expérience subit trois répétitions. Les lectures sont faites toutes les demi-heures.

\section{Expression des résultats}

Les résultats sont exprimés par la consommation doxygène (en microlitres par Insecte) mesurée pendant quatre heures.

Afin de pouvoir comparer l'influence des différents états de jeûne sur la consommation d'oxygène, nous avọns soumis les résultats à une analyse statistique appropriée. Nous avons procédé à une analyse de variance de l'ensemble des résultats obtenus chez les reines de quatre espèces de Bombus.

\section{RÉSULTATS}

L'expérimentation a comporté pour chaque espèce de Bourdon : huit lectures successives d'oxygène consommé pour chacun des 96 individus observés, répartis en 4 lots de 24 , affectés à 4 traitements (durées de jeûne) différents.

L'analyse d'homogénéité de régression consiste à étudier séparément chaque traitement en faisant pour chacun d'entre eux une estimation de la régression. La somme des quatre degrés de liberté (d. d. l.) ainsi obtenus séparément peut alors être décomposée en un degré de liberté pour la régression générale, et trois pour les écarts entre la régression spécifique et la régression générale (donc pour l'hétérogénéité). Nous avons alors le schéma suivant (tableau I).

L'analyse d'homogénéité de régression fournit, après transformation logarithmique (1), les résultats suivants (tableau II) pour chaque espèce de Bourdon.

(1) Si l'analyse avait été réalisée en données non transformées, on aurait trouvé :

- des régressions moins significatives dans tous les cas;

- des hétérogénéités similaires pour les espèces hypnorum et lapidarius, des hétérogénéités supérieures pour pratorum, et inféricures pour lucorum. 
TАвL. 1. - Analyse d'homogénéité de régression.

TAB. 1. - Homogenitätsanalyse der Regression.

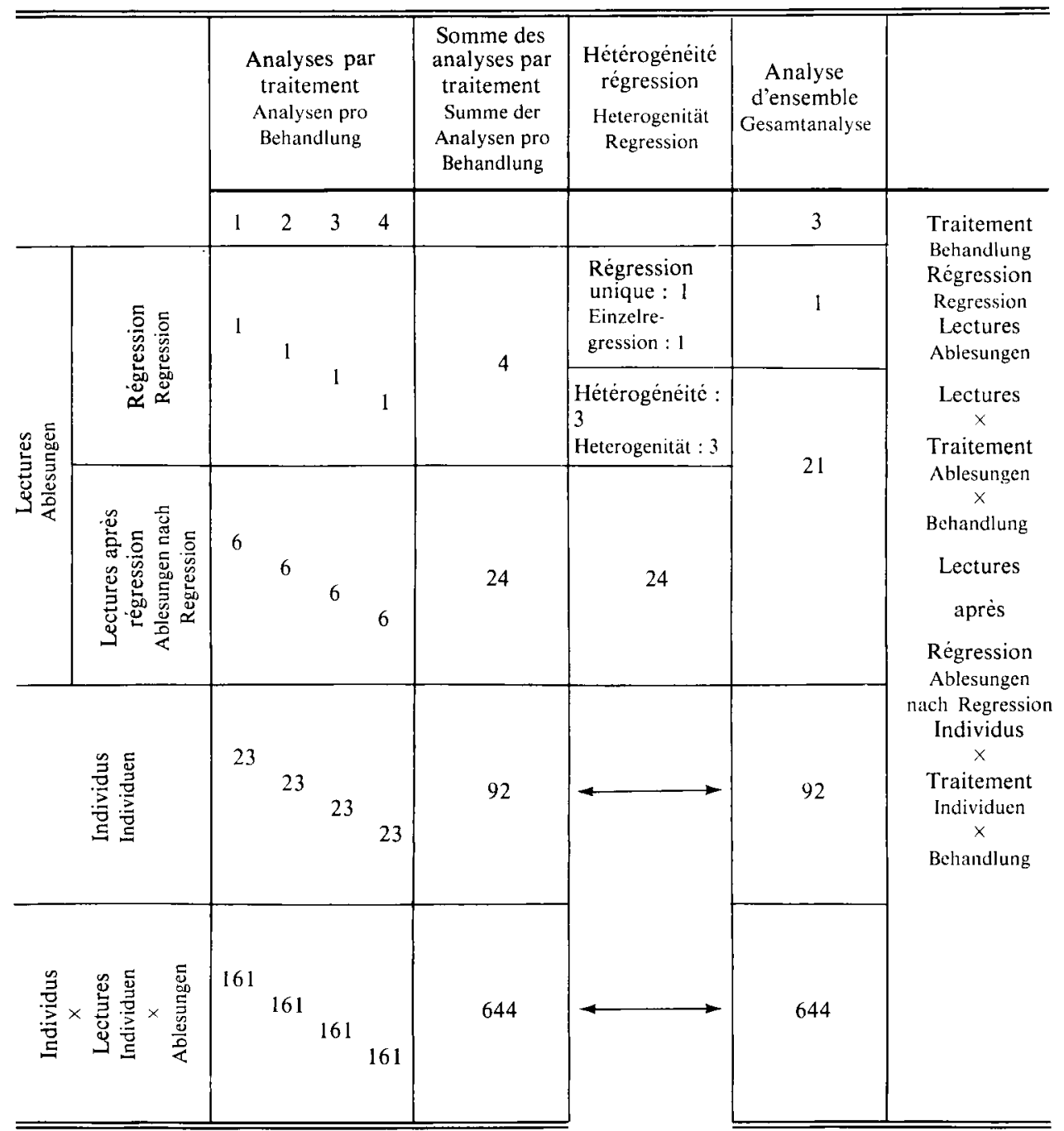

TABL. 2. - Analyse d'homogénéité de régression pour chaque espèce de Bourdon.

ТАв. 2. - Homogenitätsanalyse der Regression für jede Hummelart.

\begin{tabular}{|c|c|c|}
\hline $\begin{array}{c}\text { Espèces de Bourdons } \\
\text { Hummelart }\end{array}$ & $\begin{array}{c}F \\
\text { Régression } \\
F \\
\text { Regression }\end{array}$ & $\begin{array}{c}F \\
\text { Hétérogénéité } \\
\text { F } \\
\text { Heterogenität }\end{array}$ \\
\hline 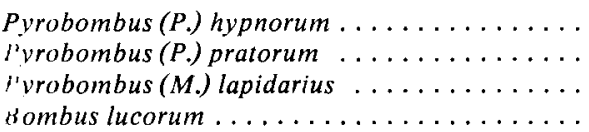 & $\begin{array}{l}69 \\
74 \\
81 \\
55\end{array}$ & $\begin{array}{l}<1 \\
> \\
1,15 \\
9,81\end{array}$ \\
\hline
\end{tabular}


Ainsi, la régression d'ensemble est très hautement significative dans tous les cas. Cependant, l'hétérogénéité des régressions ne se révèle non significative que pour les trois premières espèces. Pour la quatrième (lucorum), on trouve trois coefficients de régression très voisins et très faibles $(0,007 ; 0,008 ; 0,011)$ pour les trois premiers traitements, et un coefficient de régression élevé $(0,027)$ pour le quatrième traitement.

On peut admettre l'hypothèse de l'homogénéité des régressions, au moins pour les trois premières espèces de Bourdons étudiées.

Pour les espèces hypnorum, pratorum, lapidarius, et, dans une moindre mesure, pour lucorum, on peut donc comparer les moyennes obtenues par l'ensemble des huit lectures, soit, après transformation logarithmique : tableau III.

TABL. 3. - Moyennes de la consommation d'oxygène (en valeurs logarithmiques) en fonction du jềne chez les reines de différentes espèces de Bourdons.

TAB. 3. - Mittelwerte des Sauerstoffverbrauchs (logarithmische Werte) in Abhängigkeit von der Hungerperiode bei Königinnen von verschiedenen Hummelarten.

\begin{tabular}{|c|c|c|c|c|}
\hline $\begin{array}{l}\text { Traitements } \\
\text { Behandlungen }\end{array}$ & hypnorum & pratorum & lapidarius & lucorum \\
\hline $\begin{array}{r}1-\text { Pas de jeûne } \ldots \ldots \\
\text { unbehandelt } \ldots \ldots\end{array}$ & $\begin{array}{l}2,2985 \\
\end{array}$ & $\begin{array}{r}2,2291 \\
\quad-0,1479\end{array}$ & $\begin{array}{r}2,2844 \\
-0,0658\end{array}$ & $\begin{array}{r}2,2583 \\
-0,0801\end{array}$ \\
\hline $\begin{array}{c}2 \text { - Jeûne de } 12 \text { heures } \\
\text { Hungerperiode von } \\
12 \text { Stunden } . . \ldots \ldots\end{array}$ & $\begin{array}{r}2,2049 \\
-0,0360\end{array}$ & $\begin{array}{r}2,0812 \\
-0,0512\end{array}$ & $\begin{array}{r}2,2186 \\
-0,0359\end{array}$ & $\begin{array}{r}2,1782 \\
-0,0393\end{array}$ \\
\hline $\begin{array}{c}\text { 3- Jeûne de } 18 \text { heures } \\
\text { Hungerperiode von } \\
18 \text { Stunden ......... }\end{array}$ & $\begin{array}{r}2,1689 \\
-0,0828\end{array}$ & $\begin{array}{r}2,0300 \\
-0,0985\end{array}$ & $\begin{array}{r}2,1827 \\
-0,1209\end{array}$ & $\begin{array}{r}2,1389 \\
-0,1182\end{array}$ \\
\hline $\begin{array}{l}4 \text { - Jeûne de } 24 \text { heures } . \\
\text { Hungerperiode von } \\
24 \text { Stunden ......... }\end{array}$ & 2,0861 & 1,9315 & 2,0618 & 2,0207 \\
\hline $\begin{array}{c}\text { P.P.D.S. } \\
\text { Kleinste signifikante } \\
\text { Differenz }\end{array}$ & 0,0048 & 0,0063 & 0,0046 & 0,0053 \\
\hline
\end{tabular}

En conséquence :

- Toutes les différences entre traitements sont significatives.

- Dans tous les cas, les différences entre les traitements 1 (pas de jeûne) et 2 (jeûne de 12 heures), et entre les traitements 3 (jeûne de 18 heures) et 4 (jeûne de 24 heures) sont élevées (entre 0,0801 et 0,1479 ), tandis que les différences entre les traitements 2 et 3 sont plus faibles (entre 0,0359 et 0,0512 ), tout en restant significatives (fig. 1).

De l'analyse de variance ainsi effectuée, nous pouvons dégager des résultats pratiques représentés sur la figure 2. Chaque point des courbes exprime, après transformation logarithmique, la valeur moyenne d'oxygène consommé. 

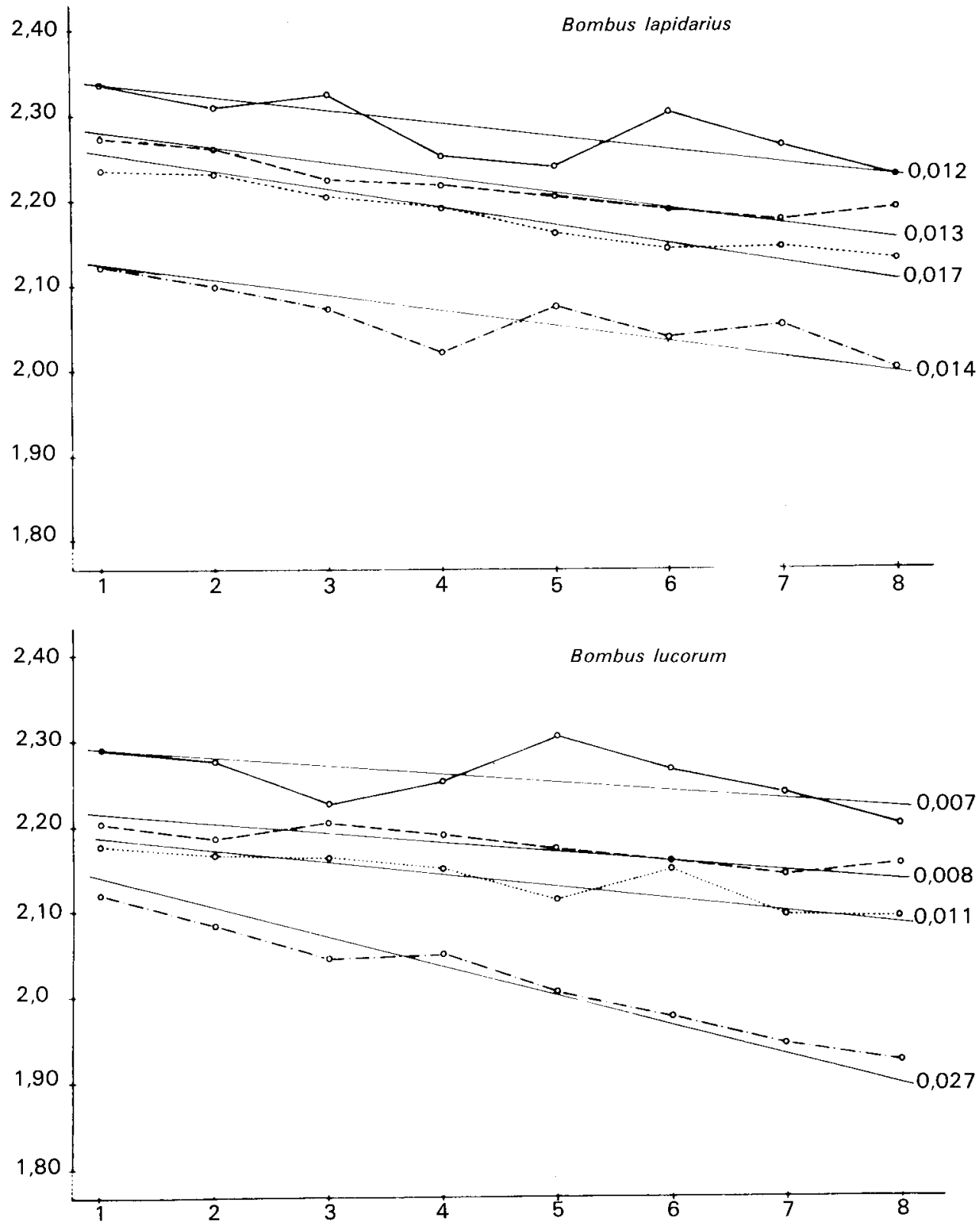

FIG. 2. - Consommation d'oxygène (en valeurs logarithmiques) en fonction de la durée de jeûne chez les reines de diverses espèces de Bombus en période d'activité.

En abscisses : numéros des lectures de la consommation d'oxygène.

En ordonnées : valeurs de la consommation d'oxygène (après transformations logarithmiques).

Reines non soumises au jeûne.
$-----\quad$ Reines soumises à un jeûne de 12 heures.
Reines soumises à un jeûne de 18 heures.

-...... . Reines soumises à un jeûne de 24 heures.

Les courbes sont assimilées à des droites dont on a calculé la pente. (Le coefficient angulaire est indiqué pour chaque droite.) 

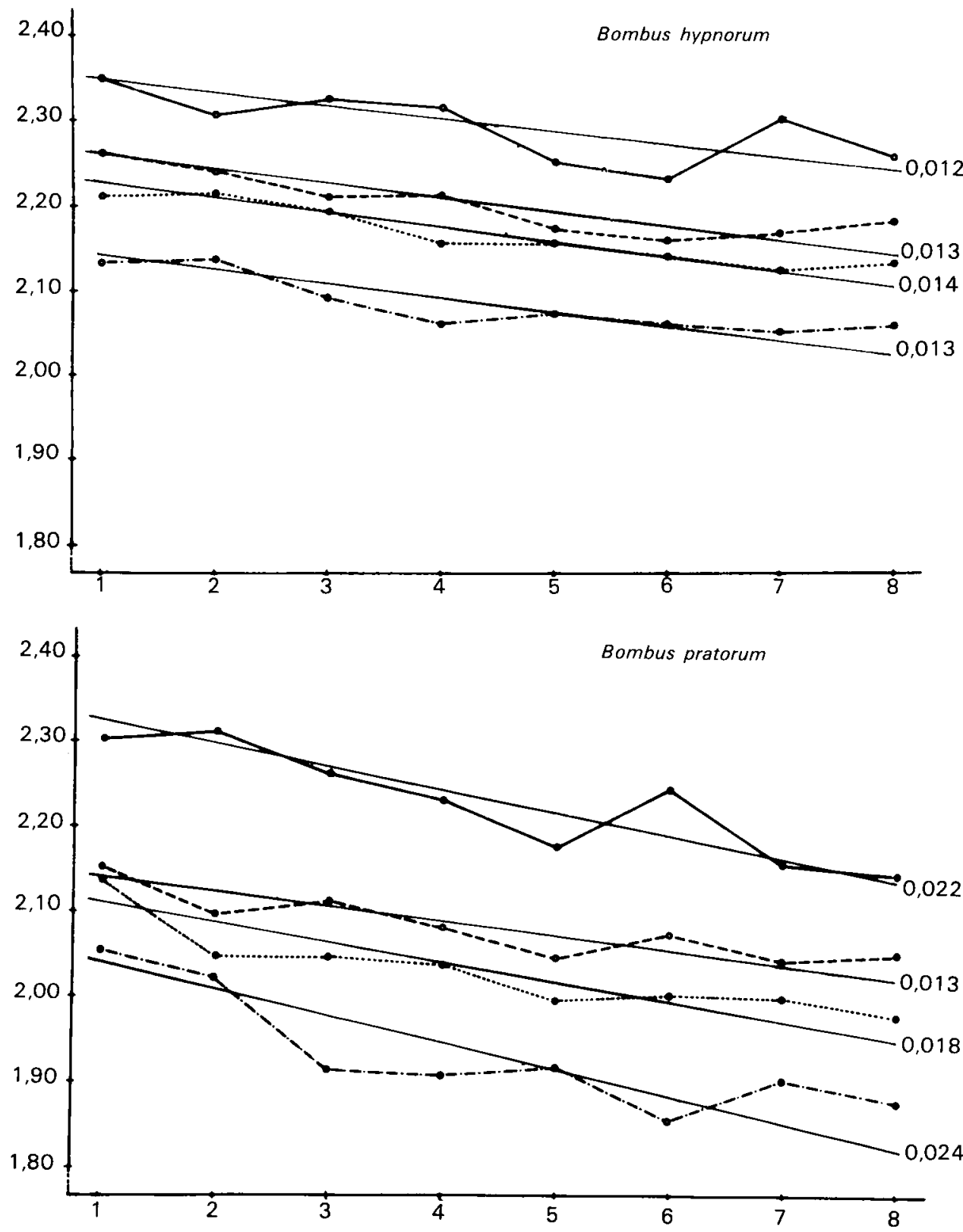

Авв. 2. - Sauerstoffverbrauch (in logarithmischen Werten) in Abhängigkeit von der Dauer der Hungerphase bei Königinnen verschiedener Hummelartensin der Aktivitätsperiode. Abszisse : Zahlen der Ablesungen des Sauerstoffverbrauchs.

Ordinate: Werte des Sauerstoffverbrauchs (nach logarithmischer Transformierung).

$\begin{array}{ll}---- & \text { unbehandelte Königinnen. } \\ ----- & \text { nach Hungerperiode von } 12 \text { Stunden. } \\ \ldots . . . . . . . & \text { nach Hungerperiode von } 18 \text { Stunden. } \\ -.-.- & \text { nach Hungerperiode von } 24 \text { Stunden. }\end{array}$

Die Kurven sind Geraden angenähert, deren Neigungswinkel berechnet wurde. Für jede Gerade ist der Neigungskoeffizient angegeben. 
Les graphiques de la figure 2 mettent en évidence les points suivants :

- Dans nos conditions expérimentales, nous constatons, chez les espèces de Bourdons étudiées, des différences dans le niveau des échanges respiratoires en rapport avec l'état de nutrition des reines.

- Un gradient apparait dans l'intensité respiratoire des Insectes en fonction de l'état de nutrition.

Les échanges respiratoires des reines alimentées normalement sont plus élevés que ceux des reines soumises à un jeûne. La durée de la période de jeûne exerce également une influence sur la respiration, celle-ci diminuant au fur et à mesure que la durée du jeûne augmente.

L'analyse de variance permet de tirer les conclusions suivantes:

- De zéro à 18 heures de jeûne, il y a proportionnalité entre le logarithme de la consommation d'oxygène et la durée de jeûne.

- La différence entre les durées de jeûne de 12 heures et de 18 heures est proportionnelle, en valeurs logarithmiques, à la différence entre zéro et 12 heures de jeûne. Les réponses, en logarithmes, de la consommation d'oxygène, sont linéaires.

- D'une façon générale, pour la durée de jeûne de 24 heures, la consommation d'oxygène est proportionnellement plus élevée que la valeur à laquelle on pourrait s'attendre si la variation entre zéro, 12 et 18 heures se poursuivait.

Les reines non soumises à un jeûne montrent des consommations d'oxygène plus inconstantes que les reines subissant une période de jeûne; les courbes présentent des oscillations de plus grande amplitude. Dans le cas des reines à jeun, les courbes respirométriques sont plus régulières, les oscillations s'atténuent nettement.

La comparaison des graphiques (fig. 2) entre les différentes espèces de Bourdon ne permet pas d'établir de relations en fonction des caractéristiques morphologiques et/ou biologiques de ces espèces.

\section{DISCUSSION}

Nos expériences montrent que les consommations d'oxygène des reines de Bombus diminuent graduellement à mesure que les durées de la période de jeûne augmentent, dans la limite de 24 heures de notre expérimentation.

Les niveaux de la consommation d'oxygène ne subissent pas une diminution progressive en fonction de la durée de la période de jeûne. Les résultats indiquent, chez les quatre espèces de Bombus étudiées, un net abaissement de la consommation d'oxygène des reines soumises à un jeûne de 12 heures par rapport aux Insectes témoins. Les reines ayant jeûné pendant 18 heures montrent des valeurs des échanges respiratoires légèrement inférieures à celles des échanges précédents. Puis, les moyennes de la consom- 
mation d'oxygène subissent une baisse un peu plus accentuée chez les reines soumises à un jeûne de 24 heures.

La diminution de l'intensité respiratoire au cours des premières heures de jeûne est, généralement, en relation avec une interruption de la digestion. La connaissance de l'évolution de cette dernière est donc fondamentale pour la compréhension des échanges respiratoires en phase d'inanition.

Nous n'avons aucune donnée sur les variations corrélatives du métabolisme respiratoire et du transit alimentaire chez les reines de Bourdons. Dans une étude comparée du transit intestinal chez les ouvrières d'Abeille (Apis mellifica L.) et de Bourdon (Bombus terrestris L.), PAIN et LACOMBE (1968) précisent les temps de transits radiologiques à travers les différentes parties du tube digestif. Chez les ouvrières de Bombus terrestris, ces auteurs constatent, dès les 15 premières minutes suivant la prise de nourriture, que l'œsophage, le jabot, ainsi que le premier pli du ventricule sont remplis du mélange nutritif opaque (miel à la gélobarine). Une heure après, le jabot et le premier pli du ventricule présentent une bonne opacification. Au bout de trois heures, presque tout l'œsophage est vide; le jabot commence à se vider. Le premier et le deuxième plis du ventricule sont pleins. A partir de quatre heures et jusqu'à six heures, l'œsophage est complètement vide; le jabot se vide également pour une grande partie. Le premier pli du ventricule présente une opacification moyenne, tandis que le deuxième pli, l'intestin postérieur et le rectum sont pleins. Ces données montrent que la durée du transit chez les ouvrières de Bombus terrestris est d'environ six heures.

Si l'on se réfère à la durée du transit intestinal (6 heures environ), telle qu'elle est mesurée par PAIN et LACOMBE, le tube digestif des reines de Bourdons devrait être vide peu de temps après la mise au jeûne. Nous estimons, toutefois, qu'il pourrait s'agir d'une durée minimale, susceptible de modifications plus ou moins importantes.

Nos conditions expérimentales (luminosité, température, texture des aliments, conditionnement, caste, âge des Insectes) étant différentes, il parait difficile d'extrapoler à partir des résultats obtenus par ces derniers auteurs.

L'évaluation du contenu intestinal est difficile à apprécier. Il semble improbable que la digestion soit terminée au début de la période de jeûne, alors que l'Insecte vient juste, dans certains cas, de finir de s'alimenter.

Lorsque les reines ont cessé d'elles-mêmes de s'alimenter, le rejet d'excréments correspondant aux derniers ingestats et au vidage du tube digestif a lieu généralement 12 à 18 heures après la dernière prise de nourriture. Pendant la période de conditionnement qui précède les expériences respirométriques, les reines commencent à déféquer le plus souvent au cours des 3 ou 4 premières heures qui suivent leur prélèvement en serre. Nous avons observé la poursuite de la défécation plusieurs heures après le début du jeûne. Il est donc possible que les fèces soient éliminées au fur et à mesure de leur formation.

Une dissection rapide permet de constater que le tube digestif des reines n'est jamais complètement vide, même au terme d'un jeûne de 24 heures. Selon WALDBAUER 
(1968), " the time required to emply the gut after cessation of feeding may be related to the speed with which food passes through the gut during feeding ".

La présence d'aliments dans l'intestin, et la poursuite des phénomènes de défécation ne permettent-elles pas de penser (BOSQUET, 1971) que les processus de la digestion ne sont pas arrêtés, mais seulement considérablement réduits?

Le ralentissement de la digestion, et, par conséquent, la diminution du niveau des échanges respiratoires, peut être passif, en relation, par exemple, avec la diminution du volume du contenu intestinal : tel est le sens des observations faites par GeLPERIN (1966) sur Phormia regina, et par Davey et Treherne (1963) sur Periplaneta americana. Mais il serait possible de concevoir également un phénomène actif, se manifestant par des modifications des sécrétions d'enzymes digestives (LANGLEY, 1966, sur Glossina morsitans; THOMSEN et MøLler, 1959, puis STRANGWAYS-Dixon, 1961, sur Calliphora erythrocephala Meig.) ou des vitesses de transit au cours du jeûne. Dans une étude sur les larves de Bombyx mori, BosQuet suppose qu'il existe un ralentissement continu des processus de la digestion au cours du jeûne, jusqu'à épuisement du contenu de l'intestin. Ce début de ralentissement serait contemporain de la modification de la courbe respirométrique observée une à deux heures après l'arrêt de l'alimentation.

Un ralentissement de la digestion au cours du jeûne semble également se produire chez les reines de Bourdons, du moins pendant la période de jeûne que nous avons adoptée. La vitesse d'élimination des fèces est susceptible de modifications importantes en fonction de facteurs quantitatifs et qualitatifs (pollen, solutions de miel à des concentrations différentes) de la nourriture ingérée.

La variabilité des durées de prise de nourriture, la nature des aliments, rendent difficile une évaluation correcte des quantités d'aliments ingérés. Par ailleurs, ainsi que le souligne LE BERRE (1963), le même ordre de précision ne saurait être exigé pour des recherches aussi différentes que celles qui traiteraient... des indices de consommation et des coefficients d'utilisation. Il devient alors nécessaire d'observer l'évolution des échanges respiratoires en dehors de toute variation du contenu intestinal, et, a fortiori, de la digestion.

L'ensemble des résultats précédents montre que la décroissance de la consommation d'oxygène au cours du jeûne chez les reines de Bourdons est un phénomène permanent, quel que soit le niveau de dénutrition atteint. A l'exception de BUCK et KeISTER (1949) et de Buck, Keister et Posner (1952) qui n'observent aucune variation au cours du jeûne chez Phormia regina Meig., les auteurs (NiEmierko et WoJtczack, 1950; ZWickY et WigglesWorth, 1956; FourChe, 1965; BosQuet, 1971) s'accordent sur l'existence d'un métabolisme respiratoire décroissant. L'évolution de la consommation d'oxygène au cours du jeûne de Drosophila melanogaster (FOURCHE) semble traduire la manifestation de processus de régulation qui réduisent d'abord brutalement le taux de la respiration pour le maintenir ensuite à une valeur relativement stable. L'existence d'une telle absorption d'oxygène conduit à l'idée d'un métabolisme de maintien nécessaire à la survie de la larve. 
Si l'on admet, selon BosQuet, l'existence d'une relation digestion-défécation, on peut émettre l'hypothèse d'une similitude entre les vitesses de diminution des échanges respiratoires et des processus de la digestion au cours du jeûne.

On peut supposer que la réduction de l'apport de métabolites à l'hémolymphe et aux organes entraîne un ralentissement des processus de synthèse. Il en résulterait une diminution de la demande en énergie et en substances, d'où une influence sur les métabolismes digestif et respiratoire. Un enchainement d'actions en retour serait susceptible d'entraîner une diminution progressive des différentes fonctions physiologiques de l'organisme de l'Insecte. C'est ainsi que BosQuet a constaté que l'intensité respiratoire et l'évacuation intestinale évoluaient de la même façon chez les larves de Bombyx mori.

Dans un tel schéma, les variations de l'intensité respiratoire répondent à la demande d'énergie créée par telle ou telle fonction, et non à la présence de matériel métabolisable. Par contre, il ne fait pas de doute que ces fonctions sont elles-mêmes régulées par la présence de certains métabolites, des contrôles neuro-endocrines, selon BosQueT, étant vraisemblables. ZWICKY et WIGGLESWORTH ont émis une hypothèse comparable : la consommation élevée d'oxygène après un repas chez Rhodnius prolixus n'a pas de rapport avec la digestion, mais serait à rapporter à la croissance qui se produit à ce moment.

Les résultats de ces expériences nous permettent de répondre, sur un plan pratique, aux questions que nous nous sommes posées. Afin que l'activité respiratoire des reines demeure plus constante, nous adoptons, pour chaque type d'expériences, des durées de jeûne variant de 12 à 18 heures. De plus, la défécation des Bourdons au cours des heures qui suivent leur prélèvement en serre impose une période de jeûne. En effet, l'élimination des égesta dans la fiole respirométrique par des Insectes n'ayant subi aucun jeûne, risque de perturber les mesures des échanges gazeux.

Considéré comme facteur expérimental, le jeûne est l'un de ceux qui perturbent le moins le métabolisme normal des Insectes. C'est un traitement ménagé, au moins à ses débuts. Il s'agit également d'un facteur naturel, car tous les Insectes, par l'intermédiaire des mues ou des facteurs climatiques, sont soumis au jeûne intermittent. Mais on peut se demander ce que représente la durée de jeûne dans l'échelle de vie de l'animal étudié; en particulier, à partir de quel moment peut-on parler de jeûne.

Dans une étude sur la thermogénèse de Bombyx mori L. et ses modifications en fonction du jeûne, Coulon (1965) montre que l'organisme des larves réagit au jeûne par une régulation du métabolisme, qui aboutit à un métabolisme de jeûne. L'établissement du métabolisme de jeûne se ferait en deux phases successives chez Bombyx:

- Disparition des calories dues au travail digestif et à l'action dynamique spécifique des aliments pendant les premières heures (fait bien établi chez les Vertébrés supérieurs).

- Mise en place d'un métabolisme d'économie à la suite d'une régulation dont le déclenchement pourrait dépendre de la valeur du taux de glycogène. 
L'utilisation de moyens d'étude différents, de méthodes biochimiques par exemple, permettrait de saisir les variations du métabolisme sous l'action de diverses durées de jeûne, et de rechercher les liaisons possibles entre les effets du jeûne et le régime, du point de vue métabolique.

Reçu pour publication en février 1979

Eingegangen im Februar 1979

\section{ZUSAMMENFASSUNG}

Die Frage des Einflusses der Ernährung auf die Intensität des Gasaustausches bei der Atmung stellt sich bei den Hummeln in der Zeit der Aktivität, besonders dann, wenn sie sich reichlich mit Reservestoffen versorgen.

Mittels der Messung des Sauerstoffverbrauches wollten wir feststellen, ob eine regelmässige Ernährung auch zu einer regelmässigen Atmungsaktivität führt, und ob eine Hungerperiode die Intensität des Gasaustausches ändert.

Die respiratorischen Messungen erfolgten mit einem Warburg-Apparat.

Unsere Untersuchungen erstreckten sich auf vier Bombus-Arten : Pyrobombus (Pyrobombus) hypnorum L., Pyrobombus (Melanobombus) lapidarius L., Bombus lucorum L., Pyrobombus (Pyrobombus) pratorum L.. Um zu einem Vergleich der verschiedenen Ernährungsstadien zu gelangen, hatten die Königinnen in diesen Versuchen dasselbe Alter und sie stammten aus demselben Volke.

Jede Messserie wurde an Hummeln in jeweils unterschiedlichen Ernährungszuständen durchgeführt :

Königinnen, die aus dem Flugkäfig entnommen und einzeln in die Behälter des Respirometers gesetzt wurden. Diese Tiere hatten im Flugkäfig freien Zugang zum Futter, aber es war nicht möglich, ihren Ernährungszustand mit Präzision festzustellen, da man die letzte Nahrungsaufnahme nicht beobachten konnte.

Königinnen im Hungerzustand. Diese Tiere wurden Hungerperioden verschiedener Dauer unterworfen : 12 Stunden, 18 Stunden, 24 Stunden.

Die Temperatur bei der Messung des Gaswechsels betrug $19^{\circ} \mathrm{C}$. Der Sauerstoffverbrauch wurde gleichzeitig bei 7 Königinnen derselben Hummelart gemessen, die sich im gleichen Ernährungszustand befanden.

Die Resultate werden als Sauerstoffverbrauch ( $\mu$ l pro Tier) angegeben, gemessen in einem Zeitraum von vier Stunden.

Für die untersuchten Bombusarten stellten wir unter unseren Versuchsbedingungen Unterschiede in der Höhe des Gasaustausches in Abhängigkeit vom Ernährungszustand der Tiere fest.

Bei normal ernährten Königinnen ist die Atmungsintensität grösser als bei Königinnen im Hungerzustand. Dabei spiélt auch die Dauer der Hungerperiode eine Rolle; die Atmung wird in dem Masse vermindert, in dem die Zeit des Hungerns zunimmt.

Zwischen einer Hungerzeit von 0 bis 18 Stunden ist der Logarithmus des Sauerstoffverbrauchs der Dauer des Hungerns proportional. Der Unterschied zwischen 12 Stunden und 18 Stunden Hunger ist proportional dem Unterschied zwischen 0 und 12 Stunden Hunger. Die dazugehörigen Werte des Sauerstoffverbrauchs, dargestellt als Logarithmus, sind linear.

Allgemein kann gesagt werden, dass der Sauerstoffverbrauch nach 24 Stunden Hunger im Vergleich 4 dem Betrag, den man nach der Variation zwischen 0, 12 und 18 Stunden erwarten müsste, erhöht ist.

Königinnen, die nicht gehungert hatten, zeigen einen viel unregelmässigeren Sauerstoffverbrauch als Königinnen nach einer Hungerzeit; die Kurven weisen viel grössere Schwankungen auf. Bei hungernden Königinnen sind die Respirometerkurven regelmässiger und die Oszillationen geringer. 
Unsere Versuche zeigen, dass sich der Sauerstoffverbrauch von Hummelköniginnen in dem Masse schrittweise verringert, in dem die Hungerperiode verlängert wurde, zumindest im Bereich der 24 Stunden unseres Versuches.

Das Niveau des Sauerstoffverbrauches zeigt jedoch keine progressive Verminderung mit der Dauer der Hungerperiode. Bei den vier untersuchten Bombus-Arten zeigte sich nach 12 Stunden Hunger im Vergleich zu den Kontrolltieren eine deutliche Senkung des Sauerstoffverbrauchs. In der nächsten Gruppe, nach 18 Stunden Hunger, zeigen die Königinnen etwas geringere Atmungswerte als die der ersten Gruppe. Nach einer Hungerzeit von 24 Stunden ist wieder eine etwas stärkere Verringerung des Sauertoffverbrauchs festzustellen.

Die Verringerung der Atmungsintensität steht während der ersten Stunden der Hungerperiode in Zusammenhang mit einer Unterbrechung der Verdauung. Die Kenntnis der letzteren ist deshalb von grundlegender Bedeutung für das Verständnis der Atmungsvorgänge in der Hungerphase.

Wenn man die Dauer der Darmpassage (etwa 6 St.) berücksichtigt, wie sie von PaIN und LACombE (1968) bestimmt wurde, sollte der Verdauungstrakt der Hummelköniginnen schon kurze Zeit nach Beginn der Hungerperiode leer sein. Wir schätzen jedenfalls, dass es sich um eine sehr kurze Zeit handelt, die ziemlich starken Schwankungen unterworfen ist. Da unsere Versuchsbedingungen (Licht, Temperatur, Zusammensetzung der Nahrung, Konditionierung, Kaste, Alter) andere waren, erscheint es uns schwierig, die Ergebnisse dieser Autoren auf unsere eigenen zu übertragen. Eine Bewertung des Darminhaltes ist schwer durchführbar. Es erscheint unwahrscheinlich, dass die Verdauung zu Beginn der Hungerperiode abgeschlossen ist, da die Tiere in manchen Fällen gerade vorher Nahrung aufgenommen hatten.

\section{RÉFÉRENCES BIBLIOGR APHIQUES}

BosQuet G., 1971. - Évolution du métabolisme respiratoire au cours du jeûne chez Bombyx mori L. - Thèse de spécialité ( $3^{\mathrm{e}}$ cycle). Univ. Cl. Bernard. Lyon. $50 \mathrm{p} .+$ fig.

Buck J. B., KeIster M. L., 1949. - Respiration and water loss in the adult blow-fly Phormia regina, and their relation to the physiological action of D.D.T. - Biol. Bull., 97; 64-81.

Buck J. B., Keister M. L., Posner I., 1952. - Physiological effects of D.D.T. on Phormia larvae. - Ann. ent. Soc. Amer., 45; 369-384.

Coulon J., 1965. - La thermogénèse larvaire de Bombyx mori L. et ses modifications en fonction du jếne. - Thèse de spécialité (Biol. anim.). Lyon. $74 \mathrm{p}$.

Davey K. G., Treherne J. E., 1963. - Studies on the crop function in the Cockroach Periplaneta. I. Mechanism of the crop emptying. - J. Exper. Biol., 40; 763-776.

FOURCHE J., 1965. - La respiration larvaire chez Drosophila melanogaster. Consommation d'oxygène au cours du jeûne. - C. R. Acad. Sci. Paris, 261; 3478-3481.

Gelperin A., 1966. - Control of crop emptying in the blowfly. - J. Insect Physiol., 12 (3); 331-345.

LANGLEY P. A., 1966. - The control of digestion in the tse tse Fly, Glossina morsitans. Enzyme activity in relation to size and nature of the meal.- J. Insect Physiol., 12; 438-448.

LE BerRe J. R., 1963. - Examen critique des méthodes d'étude de l'alimentation chez les Insectes. - In : "La nutrition chez les Peocilothermes". Journ. scient. du C.N.E.R.N.A. Paris C.N.R.S.; 249-282.

Niemierko W., WoJtczack L., 1950. - Studies on the biochemistry of the wax-moth (Galleria mellonella). III. Oxygen consumption by the larvae during starvation. Acta. Biol. Exp., 15; 79-90.

Pain J., LACOMBE J., 1968. - Données de morphologie radiologique du tube digestif d'Apis mellifica L. et de Bombus terrestris L. I. Étude comparée du transit. - Ann. Abeille, 11 (1); 15-30.

Pouvreau A., 1976. - Contribution à la biologie des Bourdons (Hymenoptera, Apoidea, Bombinae, Bombus Latr.). Étude de quelques paramètres écologiques et physiologiques en relation avec l'hibernation des reines. Thèse. Univ. Paris-Sud (Centre d'Orsay). 
StRANGWAys-Dixon J., 1961. - The relationship between nutrition, hormones and reproduction in the blowfly, Calliphora erythrocephala. - J. Exper. Biol., 38; 225.

ThOMSEN E., M $\varnothing$ LLER I., 1959. - Neurosecretion and intestinal protease activity in an insect, Calliphora erythrocephala Meig. - Nature, 183; 1401-1402.

Waldbauer G. P., 1968. - The consumption and utilization of food by Insects. - Adv. Insect Physiol., 5; 229-288.

ZWICKY K., WIGGLESWORTH V. B., 1956. - The course of oxygen consumption during the moulting cycle of Rhodnius prolixus Stäl. (Hemiptera). - Proc. Roy. entomol. Soc. London, A, 31; 153-160. 